d'où KVA totaux $=\sqrt{3.031,5^{2}+1.695,5^{2}}=3.473$ avec cès $\varphi=0,873$ et $\sin \varphi=0,487$.

Donc la variation dans le primaire est :

$$
\dot{\operatorname{Rég}}_{\mathrm{np}}=\left(\frac{3.473}{3.501}-1\right) \times 100=-0,800 \%
$$

d'où

$$
\text { Régulation }\left\{\begin{array}{l}
\mathrm{SP}=\operatorname{Rég}_{\mathrm{np}}+\operatorname{Reg}_{\mathrm{sn}}=-0,800+0,94=0,140 \% \\
\mathrm{TP}=\operatorname{Rég}_{\mathrm{n}}+\operatorname{Rég}_{\mathrm{np}}=-4,95-0,800=-5,75 \% \\
\mathrm{ST}=\operatorname{Rég}_{\mathrm{sn}}-\operatorname{Rég}_{\mathrm{tn}}=0,94-(-4,95)=\hat{+} 5,89 \%
\end{array}\right.
$$

Si nous effectuons les calculs analogues pour C. S. $=50 \%$ de $6.500 \mathrm{KVA}, \mathrm{C} . \mathrm{S} .=0$ et C. S. $=-25 \%$ de $6.500 \mathrm{KVA}$ nous trouvons les valeurs suivantes:

\begin{tabular}{|c|c|c|c|c|}
\hline $\begin{array}{c}\text { KVA du C. S. } \\
\text { en } \%\end{array}$ & $100 \%$ & $50 \%$ & 0 & $-25 \%$ \\
\hline Rég SP en $\%$. & +0.140 & +0.78 & +1.502 & +1.90 \\
Rég TP...... & -5.75 & -2.636 & +0.562 & +2.197 \\
Rég ST ..... & +5.89 & +3.415 & -0.94 & -0.297 \\
\hline
\end{tabular}

II. - Charge variable avec $\cos \%=1$ au secondaire et 2.166 KVA $\cos p=0$ avec condensateur synchrone.

Le cas de $100 \%$ de $3.000 \mathrm{KVA}$ au secondaire a dejà été vu cidessus.

\begin{tabular}{|c|c|c|c|}
\hline $\begin{array}{l}\text { KVA au } \\
\text { secondaire à } \\
\cos p=1\end{array}$ & $\begin{array}{c}100 \% \\
\text { de } \\
3.000 \mathrm{KVA}\end{array}$ & $50 \%$ & 0 \\
\hline $\begin{array}{l}\text { Rég SP en \% } \\
\text { Rég TP } \\
\text { Reg ST ...... }\end{array}$ & $\begin{array}{l}+0.140 \\
-5.75 \\
+5.89\end{array}$ & $\begin{array}{l}-0.53 \\
-5.95 \\
+5.42\end{array}$ & $\begin{array}{l}-1.28 \\
-6.23 \\
+4.95\end{array}$ \\
\hline
\end{tabular}

En effectuant les calculs de la mème manière que précédemment, nous trouvons :
III. - $3.000 \mathrm{KVA}$ à $\cos q$ variable et $2.166 \mathrm{KVA}$ au condensataur synchrone.

Pour $\cos q=1$ nous avons déjà trouvé les résultats.

\begin{tabular}{|c|c|c|c|}
\hline $\begin{array}{c}\operatorname{Cos} ? \\
\text { au Sec. }\end{array}$ & 1 & 0.90 & 0.80 \\
\hline Rég SP en $\%$ & +0.140 & +0.23 & +0.730 \\
\hline Rég TP........ & -5.75 & -5.66 & -5.15 \\
\hline Rég ST.... & +5.89 & +4.89 & +5.88 \\
\hline
\end{tabular}

Pour $\cos \tau=0,90$ et 0,80 (courant décalé en arrière de la tension), la même méthode de calculs donne le tableau suivant :

De ces résultats, nous concluons que la variation de tension entre PS s'étend au maximum de 1,90\% ̀̀ - $1,18 \%$ dans les cas extrêmes; nous en concluons que si le C. S. a son fonctionnement contrôlé par un transformateur de tension dont le primaire est branché sur le $45.000 \mathrm{~V}$., et si nous maintenons une tension constante sur le $45.000 \mathrm{~V}$., la tension sur le primaire sera dans les limites normales de fonctionnement, constante à $1 \%$ près.

Bien qu'à cause du prix très élevé on n'ait pas songé à contrôler le condensateur synchrone par un transformateur de tension $120.000 \mathrm{~V}$., nous voyons cependant que la tension sur le réseau $120.000 \mathrm{~V}$. sera pratiquement constante.

En revanche la tension sur bornes $5.500 \mathrm{~V}$. pourra être élevée à $106 \%$ de sa valeur normale et il faut que le condensateur puisse supporter cette surtension sans inconvénient.

Nous avons voulu montrer pour un cas pratique que l'on peut réaliser ces transformateurs spéciaux dans des conditions répondant aux nécessités de la régulation.

L'étude du courant de court-circuit de ces mêmes transformateurs fera l'objet d'un prochain article.

Ces transformateurs sont en cours d'exécution dans les usines de MM. Schneider et Cie et sont destinés au poste de JeanneRose du réseau de Rhône et Jura.

\title{
Considérations économiques sur l'électrification des chemins de fer ${ }^{(1)}$
}

\author{
par M. DUPIN, Ingénieur des Ponts et Chaussées
}

On a déjà beaucoup écrit au sujet de l'électrification des chemins de fer, mais surtout au point de vue technique. Je voudrais seulement ici, après avoir sommairement précisé l'état actuel de la question en France, rechercher quels sont, au point de vue économique, les principaux résultats qu'il est permis d'attendre du développement de ce nouveau mode de traction.

Quatre grands réseaux français, l'Etat, le P.-L.-M., le P.-O, et le Midi procèdent actucllement, mais dans des mesures très différentes, à l'électrification d'une partie de leurs lignes.

Le programme actuel du réseau de l'Etat est de beaucoup le moins important des quatre; il se borne, en effet, à l'électrification des lignes de la banlieue de Paris et porte sur un total d'un pcu moins de 400 kilomètres; il est vrai qu'il s'agit là de lignes ayant à assurer un trafic considérable.

Le P.-L.-M. sera vraisemblablement plus tard celui des réseaux français qư possédera le plus grand nombre de kilomètres de

(1) Extrait des Annales des Ponts et Chaussées (NovembreDécembrc 1923). lignes électrifiées; cela tient à la grande étendue de ce réseau, riche à la fois en lignes à très gros trafic et en lignes de monta-. gnes, et à ce que le Rhône, ses affluents et quelques autres cours d'eau descendant -des Alpes ou du Massif Central, lui fourniront aisément toute l'énergie qui pourra lui ètre nécessaire. La Compagnie P.-L.-M. envisage dès maintenant l'électrification de plus de 2.000 kilomètres de ligne; mais, avant de se lancer dans des travaux de cette envergure, elle désire effectuer quelques essais sur son propre réseau et, dans ce but, elle procède actuellement à l'électrification de la ligne de Culoz à Modane qui, sur une longueur totale de 140 kilomètres environ, présente un profil très varié se prêtant parfaitement aux-expériencès envisagées. L'équipement doit en être terminé dans un délai de trois ans.

L'énergie nécessaire pour assurer la traction entre Culoz et Modane sera achetée par le P.-L.-M. à la Société d'Electrochimie, d'Electrométallurgie et des Aciéries Electriques d'Ugine.

Le P.-L.-M. envisage égalemènt pour une date prochaine 1928 vraisemblablament - l'emploi de la traction électrique sur deux lignes du littoral méditerranéen : Carnoules-Vintimille et Cannes-Grasse, ce qui représente une longueur totale de 174 
kilomètres. L'énergie nécessaire serait fournie par la Société d’énergie électrique du littoral méditerranéen.

Le P.-O. et le Midi sont sensiblement plus avancés.

Le programme général d'électrification du P.-O. s'étend :

a) à la grande ligne Paris, Brive, Montauban, ainsi qu'à la ligne Brive, Figeac, Toulouse ;

b) aux transversales Saint-Sulpice-Laurière, Gannat, Périgueux, Tulle, Clermont-Ferrand; Figeac, Aurillac, Neussargues, Arvant;

c) à d autres lignes de moindre importance dans la région du Massif Central.

Ce vaste programme ne doit pas être réalisé d'un seul coup, et la Compagnie se propose comme première étape d'électrifier les 3 lignes de Paris à Brive, de Saint-Sulpice-Laurière à Gannat et de Brive à Clermont-Ferrand. Elle produira elle-même une partie de l'énergie qui lui sera nécessaire; dans ce but, elle a demandé et obtenu la concession de l'aménagement de la Haute-Dordogne et de déux de ses affluents; le Chavanon et la Rhue. Deux usines, celles de Coindre et de la Cellette, sont dès maintenant en construction sur ces affluents; une troisième usine doit ètre ultérieurement construite sur la Dordogne, près de Vernéjoux.

$D$ autre part, il est prévu que la compagnie de Paris-Orléans recevra de l'énergie de certaines usines thermiques de la région parisienne (Genevilliers-Billancourt-Vitry) et de l'usine d'Eguzon, qui vient d'ètre concédée sur la Creuse à la Société anonyme l'Union hydro-électrique; la construction de cette dernière usine est déjà commencée et sera vraisemblablement términée en 1925 .

La compagnie des chemins de fer de Paris à Orléans se propose d'assurer le transport de l'énergie jusqu'aux sous-stations alimentant les conducteurs de contact au moyen de lignes à 150.000 volts et à 90.000 volts. Elle a déjà demandé la concession d'une ligne à 150.000 volts et de 2 lignes à 90.000 volts entre Eguzon et Paris. Les demandes de concession des autres lignes de transport seront présentées au fur et à mesure des besoins.

La Compagnie des chemins de fer du Midi est de beaucoup celle dont les travaux d'électrification sont les plus avancés. Il y a d'ailleurs de nombreusès années que le Midi est entré dans cette voie et, avant la guerre, il comptait déjà 170 kilomètres de lignes électrifiées; il est vrai qu'il lui a fallu ultérieurement transformer tout ce qui avait été fait à cette époque : les premières lignes avaient été établies en effet de façon à utiliser du courant monophasé, mais, depuis, une décision ministérielle, intèrvenue à la suite des travaux d'un comité d'études constitué en 1918, a spécifié que tous lés réseaux francais ne devraient utiliser que du courant continu à la tension de 1.500 volts (ou exceptionnellement de 3.000 volts lorsque les conditions spéciales le justifieraient) toutes les locomotives d'un réseau quelconque devant pouvoir circuler et s'alimenter en énergie sur toutes les lignes électrifiées à 1.500 volts en France.

Les principales raisons qui ont conduit au choix de ce courant sont :

Pour le choix du type continu (1):

(1) Si le courant continu à haute tension peut être considéré dans l'état actuel de la science électrique comme un des meilleurs, beaucoup disent le meilleur, des courants de traction, s'il a été également adopté par la Belgique, l'Angleterre, une partie des Etats-Unis, etc... il ne jouit cependant pas de la faveur universelle. La Suisse Jui préfère le monophasé qu'elle utilise depuis un certain temps déjà et dont elle se déclare fort satisfaite ; la Suède, la Norvège, l'Allemagne font de même.

L'Italie paraît être le seul pays qui ait adopté le triphasé, mais le fait qu'elle commence à expérimenter sur certaines lignes d'autres systèmes et en particulier celui de la traction par courant continu, semble indiquer qu'elle n'a pas eu particulièrement à se louer de l'emploi du triphasé. a) Le fait que ce courant paraît être celui qui apporte le moins de trouble au fonctionnement des lignes télégraphiques ou téléphoniques;

b) La possibilité d'utiliser pour l'alimentation des sous- stations de traction n'importe quelle distribution d'énergie, quelle que soit la tension et la période de son courant, car il est toujours facile de transformer un courant quelconque en continu.

Pour le choix de la tension 1.500 volts :

La pössibilité d'utiliser indifféremment pour l'alimentation des locomotives électriques un conducteur aérien ou un troisième rail (1). Il a été spécifié que tous les locomoteurs devraient ètre équipés de façon à pouvoir circuler indifféremment sur les voies munies de ligne de prise de courant aérienne ou de $3^{\mathrm{e}}$ rail.

Les travaux d'électrification de lá compagnie du Midi sont actuellement limités à la partie de son réseau usitée à l'ouest de Toulouse (2), et les lignes dont on prévoit l'électrification dans cette zone sont celles de Bordeaux, Bayonne, Hendaye, et celles de la région pyrénéenne.

Pour la production de lénergie, le Midi disposera de 5 usines importantes : d'une part, les usines de Soulon ef d'Eget, construites, la première avant et la seconde pendant la gucre: d'autre part, les usines de la vallée d'Ossau : Artouste, Miegebat, Houral, dont les travaux sont en cours d'exécution.

Le transport de lénergie nécessaire à l'alimentation des sousstations sera assuré par deux réseaux, l'un à 150.000 volts s'étendant jusqu'à Toulouse et Bordeaux, l'autre à 60.000 volts qui suivra en principe le tracé des roies ferrées électrifiées. La construction du réseau à 150.000 volts est à peu près terminée, celle du réseau à 60.000 volts se développe parallèlement à l'électrification des lignes de chemin de fer. La traction électrique fonctionne dès maintenant sur plusieurs lignes ou tronçons de lignes et la compagnie du Midi estime être en mesure de l'adopter complètement :

En 1924, sur les lignes :

Toulouse, Montréjeau, Pau, Dax; Montréjeau, Luchon; Lannemezan, Arreau; Tarbes, Bagnères ; Lourdes, Pierrefitte;

En 1925, sur les lignes :

Bordeaux, Irun ; Lamothe, Arcachon;

En 1926, sur les lignes:

Pau, Bedous, Frontière; Buzy, Laruns ; Porlet Saint-Simon, Ax-les-Thermes, Frontière.

On voit, d'après ce qui précède, que les trois compagnies, du P.-L.-M., du P.-O. et du Midi vont avoir recours, pour se procurer l'énergie nécessaire aux premières lignes qu'elles électrifient, à des solutions fort différentes : le P.-L.-M. se propose de l'acheter à des sociétés productrices; le Midi, au contraire, construit des usines capables de satisfaire à l'ensemble de ses besoins; quant au P.-O., il a adopté un système mixte, combinaison des deux précédents.

De pareilles différences de méthode peuvent surprendre. Elles ne sont cependant pas inexplicables, car bien des considérations entrent ici en jeu et il n'est pas très étonnant que l'importance

(1) Au-delà de 1.500 volts, l'emploi du troisième ratil ne semble plus possible.

(2) A la suite de l'électrification de cette zone, le Midi envisage celle des lignes situées danslles Pyrénées Orientales et sur le versant sud-ouest du Massif Central. 
relative que les diverses compagnies attachent à chacune d'elles, el par suite les conclusions auxquelles elles aboutissent, ne soient pas toujours les mêmes.

On conçoit fort bien, par exemple, qu'une compagnie se propose de produire elle-mème la totalité de l'énergie qui lui sera nécessaire dans des usines lui appartenant et construites spécialement à cet elfet (1); elle y trouvera, entre autres avantages, celui de conserver pour elle le bénéfice que se réserveraient les vendeurs d'énergie. On peut objecter, il esL vrai, que les Compagnies de chemins de fer n'ont pas coulume d'exploiter ellesmêmes des mines pour en extraire le charbon dont elles ont besoin, bien que des raisons du même genre puissent être invoquées en faveur de cetle exploilation, mais l'on doit reconnaître que l'aménagement et surtout l'exploitation d'une usine hydroélectrique et celle d'une mine ne sont pas choses comparables et que tel qui reculerait devant l'une de ces entreprises peut très bien exécuter l'autre. Il convient cependant de remarquer que la méthode qui consiste pour une compagnic à produire elle-même la totalité de son énergie doit normalement l'entraîner à réaliser des aménagements considérables, et cela pour deux raisons : la premiere, c'est qu'il faut que la puissance disponible pendant lns mois d'éliage ou les années sèches soit suffisante pour lui permettre d'assurer son service; la seconde, c'est que, pour eviter une spécialisation exagérée dont le rendement serait médiocre, il esl bon qu'une certaine fraction de l'énergie soit fournie par des usines dont la fonction principale ne soit pas "l'alimentation des chemins de fer. C'est pourquoi une compagnie qui voudrait n'avoir recours à aucune source d'énergie extérieure serait conduite à construire des usines dont la puissance normale excéderait sensiblement ses besoins et obligée ensuite de rechercher des débouchés pour ses excédents d'énergie.

En sens contraire, on conçoil également très bien qu'une compagnie préfère acheler à quelques gros producteurs ou distributeurs l'énergie dont elle a besoin pour assurer la traction sur ses lignes. Elle se libc̀re ainsi du souci d'aménager des chutes, ce qui exige toujours des capitaux considérables, et de celui de les exploiter. En outre, si le distributeur auquel elle s'adresse possède en même temps que des usines hydrauliques des usines thermiques, elle peut y voir à bon droit une sécurité supplémentaire. Pareille founiture peut d'ailleurs se faire dans des conditions très intéressantes pour les Compagnies, car elles sont certainement d'excellents elients auxquels on peut faire des conditions avantageuses et cela non seulement parce que les quantités d'énergie qu'elles ạbsorbent sont considérables, mais encore parce que le fait pour un distributeur de compter parmi sa clientèle une compagnie de chemins de fer lui permettra très souvent d'améliorer sensiblement ses conditions d'exploitation. Les réseaux auront en effet une excellente durée d'utilisation de la puissance, très supérieure à celle que l'on oblient en général dans les distributions. Cela tient : $a$ ) à la compensation qui s'effectuera entre le trafic des différentes lignes dès qu'une partie importante des réseaux sera électrifiée; $b$ ) à la possibilité de modifier les horaires de cerlains trains (trains de marchandises surtout) en tenant comple des conditions de fonctionnement dont s'accommoderont le mieux les usines génératrices, de façon à atténuer les pointes journalières qui constiluent une si lourde sujétion pour les exploitants; c) à l'emploi, déjà envisagé par quelques réseaux, d'une certaine quantilé de matériel à accumulateurs, chargés pendanl les heures creuses.

(1) Elle n'aurail même pas la possibilité d'envisager une autre solution s'illne se tronvait personne pour lui offrir de l'alimenter en energic.
Entre ces deux solutions extrêmes - production par les compagnies exclusivement, achat de la totalité de l'énergie à des distributeurs, - il y a place pour une infinité de solutions intermédiaires dont l'une des variètés consiste dans la participation des Compagnies à des aménagements grandioses, telle la participation du P.-L.-N. à l'aménagement du Rhône. Il est vraisemblable que c'est moins à l'adoption exclusive d'un de ces systèmes qu'à la combinaison de systèmes différents qu'aboutiront finalement toutes les compagnies de chemins de fer.

L'électrification des chemins de fer ou, d'une façon plus précise, celle d'une ligne déterminée, n'est pas forcément une opération avantageuse, et peut même apparaître comme absolument contre-indiquée. Pour s'en convaincre, il y a deux choses dont il importe de se bien rendre compte. Ce sont : le coût de l'équipement électrique des voies ferrées et l'importance des pertes d'énergie en ligne

Le coût de l'équipement électrique est évidemment fonction de la nature de la ligne de chemin de fer considérée : il varie süivant que la ligne est à voie simple, double, quadruple..., suivant que la puissance nécessaire est plus ou moins grande sur le tronçon considéré, suivant la vitesse prévue, car lorsque les organes de prises de courant doivent se déplacer avec une vitesse de 130 kilomètres à l'heure sur le conducleur de contact, il faut évidemment que celui-ci soit établi dans des conditions offrant des garanties mécaniques particulières.

Il n'est donc pas facile d'indicuer avec exactitude, et en dehors de tout cas particulier, le prix de revient de l'équipement électrique des voies ferrées. On peut du moins en indiquer l'ordre de grandeur, et c'est ce que je vais essayer de faire.

Si l'on considère uniquement l'équipement électrique proprement dit d'une voie ferrée existante, c'est-à-dire l'installation du conducteur de prise (tróisième rail ou ligne aérienne) de courant continu à 1.500 volts et l'éclissage électrique des rails de roulement, par lesquels se fait le retour du courant, sans par conséquent se préoccuper des organes d'alimentation (lignes à haute-tension, sous-stations de transformation...), on peut admettre que pour une ligne de chemin de fer à double voie le coût de cette installation est actuellement voisin de 200.000 francs par kilomètre, ce prix pouvant d'ailleurs ètre sensiblement: dépassé s'il est nécessaire de faire subir des remaniements importants aux installations antérieures, en particulier aux lignes téléphoniques et télégraphiques.

Il est plus difficile d'évaluer le prix des sous-stations de transformation du courant alternatif en continu 1.500 volts, car ici les dispositions adoptées varient fortement d'un eas à un autre. L'intervalle moyen entre les sous-stations est d'une vingtaine de kilomètres; il peut exceptionnellement descendre à dix ou dépasser 30 . Leur armement, donc leur prix, dépend essentiellement du trafic prévu sur les lignes qu'elles doivent desservir. Revenant à la ligne de chemin de fer à double voie déjà considérée, on aura une idée approchée des charges résultant de la construction de ces sous-stations en tablant sur une dépense de 100.000 francs par kilomètre de ligne à double voie.

Groupant ces deux dépenses : équipement des voies, sousstations de transformation en courant continu 1.500 volts, on arrive à un total d'environ 300.000 francs par kilomètre (1). Il resterait, pour être complet, à tenir compte du coût des lignes

(1) Pour une ligne à voie simple, la dépense serait naturellement plus faible, mais non pas de moitić. Au contraire, pour une ligne à voie quadruple, elle serait plus élevée, mais resterait inférieure au double, a condition, bien entendu, que toutes choses soient égales par ailleurs. Si la ligne à 4 voies était établie dans des con- 
à haute tension et des postes de transformation qui leur correspondent. Mais là toute évaluation moyenne devient absolument dépourvue de sens par suite de la différence des situations (1). Et d'ailleurs, mème pour un réseau délerminé, la répartition par kilomètre de voie ferrée des dépenses correspondant à la haute tension resterait fort difficile. En effet, les lignes à 150.000 volts, à 90.000 volts, à 60.000 volts, etc... serviront toutes plus ou moins à transporter de l'énergie pour le compte de tiers; les compagnies percevront en échange un péage et les"sommes qu'elles encaisseront ainsi diminueront d'autant leurs propres frais; mais on ne peut encore évaluer avec précision l'importance de ces péages. On ne peut pas davantage dire exactement dès maintenant jusqu'où s'étendra l'électrification des réseaux, c'est-àdire le nombre de kilomètres de voies qui seront appelés à utiliser l'énergie transportée par ces lignes à très haute tension. Pour ces raisons, la répartition par kilomètre de voie ferrée des dépenses correspondant à la haute tension reste encore tout à fait indéterminée.

Quoi qu'il en soit, on voit d'après ce qui précède que, tout compris (hormis les usines productrices dont il sera tenu compte pour l'évaluation du prix du courant), il n'est pas invraisemblable d'admettre que les travaux d'électrification d'une ligne de chemin de fer à double voie reviennent à près de 350.000 francs par kilomètre (2).

L'intérêt annuel de cette somme, calculé à 7,5\%, est de 26.250 francs. Si à ce chiffre l'on ajoute :

a) les dépenses de matériel pour entretien et réparation;

b) l'amortissement, très difficile à évaluer, parce que l’on manque encore d'expérience en la matière, et qu'il doit d'ailleurs varier fortement suivant les conditions d'aménagement ( $3^{e}$ rail ou conducteur aérien) et d'exploitation de cháque ligne, on voit que le total des charges annuelles doit être de l'ordre de 30.000 francs par kilomètre.

Cela suffit à prouver que sur les lignes à très faible trafic l'électrification n'est pas, - à moins de raisons spéciales (3) -

ditions particulièrement soignées, sơn coût pourrait dépasser le double du chiffre indiqué plus haut. C'est ce qui a lieu par exemple pour la ligne à 4 voies de Paris à Orléans. Le P.-O. a tenu, en raison de l'importance du trafic qu'elle doit assurer et des vitesses qui y seront réalisées, à l'équiper dans des conditions offrant des garanties toutes spéciales. C'est ainsi que les 4 voies devant être munies d'une ligne de contact aérienne, 2 d'entre elles seront en plus munies d'un $3^{e}$ rail. Aussi le coût de l'équipement atteindrat-il sur cette section un prix particulièrement élevé ; il paraît devoir être voisin de 700.000 francs par kilomètre.

(1) Les lignes à 150.000 volts du P.-O. sont établies de façon à pouvoir fonctionner plus tard à 220.000 volts ; celles du Midi, non. Sur le Midi, les lignes à 60.000 volts sont très souvent établies dans les emprises du chemin de fer et portées par les mêmes supports que les lignes à 1.500 volts. Sur le P.-.O. au contraire, les lignes à 90.000 volts sont situées hors des emprises et entièrement distinctes des autres, etc...

(2) Si l'on se borne, comme ici, à envisager le problème dans son ensemble, il ne paraît pas possible de serrer cette évaluation de très près; les prix sont encore trop instables, et le coût des travaux varie trop d'une ligne à une autre. Pour pouvoir préciser, il faudrait étudier l'électrification d'une ligne déterminée, réalisée à une époque également déterminée, ce qui, pour une vue d'ensemble, n'offrirait qu'assez peu d'intérêt.

Il est à remarquer que le chiffre indiqué ci-dessus s'applique à la voie courante. Pour évaluer le coût de l'équipement d'une ligne, il ne suffirait donc pas de multiplier ce chiffre par la longueur de la ligne. Il faudrait encore tenir compte de l'équipement des voies de manœúvre ou de garage.

(3) Comme exemple de cas où des raisons spéciales interviennent, je citerai colui d'une ligne à faible trafic entourée de lignes dont l'électrification est décidée ; on aura généralement intérêt à étendre l'électrification à cette ligne pour des raisons d'homogénéité d'exploitation. à envisager. Soit, en effet, une ligne sur laquelle circulent seulement 8 trains par jour ( 4 trains montants -4 trains descendants), c'est-à-dire $8 \times 365=2.920$ trains par an. Les $30.000 \mathrm{fr}$. répartis entre ces 2.920 trains fonl un peu plus de 10 francs par kilomètre pour chacun d'eux. Il en résulte que la charge correspondant aux travaux d'électrification se trouve, à elle seule, être du même ordre que l'ensemble des frais de traclion ef d'entretien dans le cas de iraction à la vapeur (1).

Cherchons maintenant à évaluer l'importance des pertes d'énergie. Prenons, par exemple, un kilowall-heure produit par les usines de la vallée d'Ossau de la Compagnie des chemins de fer du Midi. La fraction de celte énergie qui sera effectivement utilisable pour la traction dépendra du rendement des intermédiaires. Ces rendements atteignent sensiblement :

Pour le transformateur de départ qui élève la tension

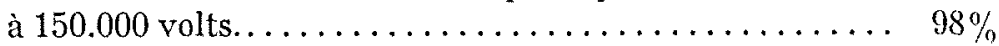

Pour la ligne à 150.000 volts $(2) \ldots \ldots \ldots \ldots \ldots \ldots \ldots 2 \%$

Pour le transformateur 150.000 volts -60.000 volts . . $98 \%$

Pour la ligne à 60.000 volts $(2) \ldots \ldots \ldots \ldots \ldots \ldots \ldots . \ldots \ldots$

Pour la ligne de travail $(2) \ldots \ldots \ldots \ldots \ldots \ldots \ldots \ldots . \ldots \ldots$

Pour la sous-station 60.000 volts -1.500 volts con-

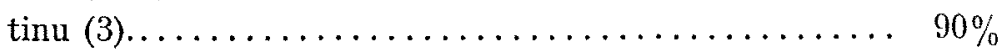

Si l'on fait le produit de toutes ces quantités, on obtient pour le rendement de l'ensemble le chiffre de 0,676. Autrement dit, la machine ne reçoit que la fraction 0,670 de chaque $\mathrm{kw}$. heure fourni par l'usine et, comme le rendement du locomoteur lui-mème n'est que de $85 \%$ à un $\mathrm{kw}$. heure pris aux bornes de l'usine, il ne correspond qu'à peine $0,6 \mathrm{kwh}$. effectivement utilisable à la jante des roues.

Ceci posé, on conçoit comment il est possible - tout au moins en première approximation — d'étudier les conséquences financières de l'électrification d'une ligne. On connaît le trafic actuel de cette ligne et la consommation de charbon correspondante; on en déduit avec une certaine approximation son trafic et la consommation de charbon pendant les années qui viennent. Cette évaluation servira de base au calcul - car c'est un principe constant que les installations électriques doivent être basées non point sur le trafic actuel d'une ligne, mais bien sur un trafic sensiblement supérieur que l'on prévoit devoir être alteint dans un certain délai. Il en résulte que pour établir le bilan économique d'une électrification, il convient de tenir comple des augmentations de trafic réalisables jusqu'au moment où les installations faites auront besoin d'être renforcées.

De la connaissance du trafic il est relativement facile de déduire la quantité d'énergie électrique nécessaire pour l'assurer. On sait en effet qu'il faut environ 20 watts-heure (4) par tonne kilométrique remorquée; soit $\mathrm{N}$ le nombre de kwh. nécessaires dans l'année. Cela correspond, le rendement de l'ensemble des organes de transport et d'utilisation étant de $0,6, \dot{a} \frac{\mathrm{N}}{0,6}$ kwh. pris aux bornes des usines. Si l'énergie est d'origine hydraulique

(1) Dans le rapport du Conseil d'administration du P.-O. pour l'année 1921, l'ensemble des f'rais de traction, d'entretien et de réparation des machines, tenders èt wagons est évalué à $11 \mathrm{fr} .50$ par kilomètre de train.

(2) Chiffre moyen, le rendement étant fonction de la longueur de ligne utilisée.

(3) Le réndement varie suivant le système de transformation adopté.

(4) Ce chiffre représente, comme l'indique la suite, de l'énergie disponible à la jante des roues. Il n'a rien d'absolu el est essentiellement fonction des conditions d'exploitation. 
et si le prix de revient du kwh. aux bornes de ces usines alteint 0 fr. 06, la dépense correspondante est de $\frac{\mathrm{N}}{0,6} \times 0,06=\frac{\mathrm{N}}{10}$ francs.

On sera donc réduit à comparer : d'un côté, les dépenses de charbon correspondant à la traction à vapeur; de l'autre, la somme : a) des $\frac{\mathrm{N}}{10}$ francs représentant le coût de l'énergie électrique; $b$ ) des dépenses d'intérêt, d'amortissement et de réparations correspondant aux installations électriques. Mais il ne faudrait pas s'en tenir là, car une pareille comparaison serait lout à fait incomplète. Tout d'abord, elle ne fait pas intervenir le coût des locomolives elles-mêmes; on admet, il est vrai, en pratique, qu'il n'y a pas lieu d'en tenir compte; que, si les machines électriques coûtent à peu près deux fois plus cher que les locomotives à vapeur, elles peuvent faire un service double et qu'il y a là compensation; l'expérience montrera ce qu'il y a de vrai dans cetle approximation sans doute très grossière. En second lieu, et ceci est plus grave, elle ne tient pas compte des nombreux avantages accessoires qu'offre l'électrification. Ces avantages seront énumérés plus loin et l'on verra qu'ils ne se prêtent guère à une évaluation numérique. D'ailleurs, même si avec une extrême habileté l'on parvenait à chiffrer de façon précise l'effet des avantages accessoires auxquels il vient d'être fait allusion, il ne faudrait pas s'illusionner sur le résultat, car tous les calculs renferment une cause d'erreur ou plutôt d'incertitude qui fait que si leur résultat peut ètre considéré comme exact à un instant donné, il a les plus grandes chances de ne plus l'être quelques mois ou quelques années plus tard. Cette cause d'erreur c'est la variation des prix, et de celui du charbon en particulier. Qui pourrait dire ce que seront ces variations durant la quinzaine d'années qui correspondra à la réalisation du premier programme d'électrification envisagé ? Sans doute on peut bien espérer qu'il y aura dans l'ensemble un certain parallélisme dans les variations à venir; que le prix du charbon, le taux de l'intérêt, le prix de la main-d'œuvre, celui de l'appareillage électrique augmenteront ou diminueront ensemble, de telle sorte que le résultat de la comparaison ne soit pas trop faussé. C'est vrai, dans une certaine limite, mais quelle est exactement cette limite?

Si incertaines que soient les évaluations qu'il est possible de faire, il est cependant bon d'avoir une idée de l'importance des économies accessoires que l'électrification permettra de réaliser. Les estimations les plus raisonnables conduisent à penser qu'elles sont de l'ordre de 0 fr. 75 le kilomètre de train, ce qui est loin d'être négligeable.

J'ai supposé plus haut que l'énergie était d'origine hydraulique. Ce n'est point qu'on ne puisse concevoir une électrification basée sur l'emploi d'énergie d'origine thermique et, en fait, ce système est déjà adopté depuis longtemps sur certaines de nos lignes el c'est le seul qui soit possible dans les pays manquant de houille blanche, comme l'Angleterre. Parmi les raisons que l'on peut invoquer en sa faveur, figure ce fait que les grosses centrales peuvent employer du charbon de moins bonne qualité, donc moins cher que celui que brûlent les locomotives, et ont un rendement très supérieur à celui de ces machines. Il n'en est pas moins vrai que ceux de nos réseaux qui s'électrifient dès maintenant en grand, c'est-ì-dire le Midi, le P.-O. et le P.-L.-M., paraissent, dans les conditions acluelles, avoir un très gros intérêt à utiliser de l'énergie d'origine hydraulique plutôt que thermique. Considérons, en effel, un même réseau ferré que nous supposerons électrifié el comparons les résultats de cette électrification en supposant l'énergie produite par des usines hydrau- liques et par des thermiques ; les dépenses correspondant à l'équipement électrique des voies, à la construction, à l'entretien des lignes de transport et des postes de transformation, etc... sont les mêmes dans les deux cas; ce qui varie, c'est le prix du kilowatt-heure aux bornes de l'usine génératrice. Or, pour de l'énergie d'origine thermique on ne peut guère compter, même en se plaçant dans de très bonnes conditions d'exploitation, que le prix de revient pour l'un quelconque des 3 réseaux considérés ci-dessus, descendrait en moyenne au-dessous de 13 centimes par kwh., le charbon étant supposé valoir 100 francs la tonne, et il existe de nombreuses usines hydro-électriques susceptibles de leur fournir de l'énergie à un prix sensiblement inférieur. La différence est assez importante pour que, même si le prix du charbon vient à redescendre notablement au-dessous de $100 \mathrm{frs}$ la tonne, l'avantage paraisse susceptible de se maintenir généralement en faveur de l'énergie d'origine hydraulique.

Cela ne veut point dire que le recours à des centrales thermiques ne puisse être envisagé en aucune façon. En premier lieu, l'énergie d'origine hydraulique étant assez inconstante, les centrales thermiques interviendront toujours utilement pour suppléer à ses défaillances. D'autre part, dans toute la partie septentrionale de la France, l'énergie électrique de source hydraulique n'arrive pas actuellement; l'y amener conduirait d'ailleurs, en raison de la longueur du transport, à une majoration sensible du prix de revient du kilowatt-heure; par contre, cette région dispose de larges ressources en charbon, et des centrales, établies sur le carreau des mines et utilisant un combustible de médiocre qualité et intransportable, peuvent arriver à y produire des kwh. particulièrement bon marché. Ces raisons font que si l'on se proposait prochainement d'électrifier des lignes de chemins de fer dans le Nord de la France, on serait très vraisemblablement amené à y envisager l'emploi d'énergie d'origine thermique.

Mais il est peu probable que dans un avenir très rapproché l'électrification des chemins de fer prenne dans le Nord et dans l'Est une grande extension; des considérations de défense nationale y font à elles seules obstacle : les lignes de transport d'énergie électrique, les postes de transformation, etc... sont en effet des objectifs très faciles pour l'aviation de bombardement et un ou deux projectiles bien placés suffiraient à interdire pour longtemps et d'une façon complète la circulation des trains électriques dans toute une région. Aussi paraît-il nécessaire, dans les conditions actuelles, que la plupart des lignes du Nord et du Nord-Est conservent leur système de traction à vapeur, et que les compagnies qui desservent ces régions continuent à utiliser et à renouveler leur stock de locomotives et leur effectif de mécaniciens et de chauffeurs.

S'il n'est pas toujours avantageux d'électrifier, il y a cependant de très nombreux cas dans lesquels l'électrification parait présenter un intérêt réel.

Et, tout d'abord, il existe deux circonstances dans lesquelles cet intérêt est évident et incontestable :

a) Certaines gares-terminus, à Paris en particulier, sont sur le point d'atteindre leur limite de trafic. Comme on ne peut songer à exproprier des surfaces considérables de terrains bâtis en vue de leur extension, la sitnation serait fort embarrassante si l'électrification n'apportait à ce problème une solution très satisfaisante : l'électrification des lignes existantes entrainera une augmentation très notable du rendement des quais actuels, grâce à l'accélération des démarrages, à l'emploi de trains de banlieue symétriques (automotrices en tête et en queue) évitant 
les manceuvres de machines, etc... Cela suffirä pour quelque temps et le jour où la nouvelle capacité de trafic sera elle-mème sur le point d'être atteinte, l'emploi de la traction électrique permettra. sans grande difficulté, la construction au-dessous de gares et lignes actuelles de gares et lignes souterraines; autrement dit, elle permettra de réaliser en hauteur une extension quasi impossible en largeur.

b) Le second cas est celui des lignes de chemins de fer à créer en terrain accidenté. On sait que la traction électrique s'accomode de rampes beaucoup plus considérables que la traction à rapeur, d'où la possibilité, dans l'étude d'une ligne qui doit ètre ejlectrifiée, de prévoir un tracé plus raide et d'un prix de revient plus faible par suite de la diminution de sa longueur, de la suppression des souterrains, etc... que s'il s'agissait d'une ligne pour traction à vapeur. L'économie réalisable de ce fail pourra dépasser considérablement l'augmentation de dépense exigée pour l'équipement électrique des voies.

Ces deux cas, pour si intéressants qu'ils soient, sont cependant exceptionnels en France. Le nombre des gares-leminus atleignant leur limite de trafic est limité, el notre réseau ferré, déjà très étendu, ne s'accrô̂t plus que lentement. Si l'électrification des chemins de fer devait se borner là, elle n'offrirait cqu'un assez faible intérèt. Voyons donc quelles peuvent ètre, dans le cas le plus général, les avantages qu'elle est susceptible de procurer.

Ils sont multiples; l'énumération ci-dessous, sans prétendre ètre complète, en domera une idée :

a) Le premier est évidemment l'économie du charbon, la plus grande partie de l'énergie devant être fommie par des usines hydro-électriques. Je n'insiste pas et ferai simplement remarquer que, le trafic des chemins de fer croissant régulièrement d'une année à l'autre en période normale, celte économie de charbon doit aller elle-mème en croissant.

b) A l'économie du charbon proprement dile se raltache celle des dépenses accessoires à l'emploi de ce combustible, dépenses qui correspondent au transport du charbon jusqu'aux dépôts; à son déchargement et à son chargement; à l'alimentation en eau des dépôts et des gares, etc... (1).

c) L'économie de main-d'œuvre, tant dans le personnel nontant sur les machines que dans celui des dépòts.

Certains trains, en effet, pourront n'être conduits que par un seul mécanicien, par exemple les trains de banlieue qui uliliseront des automotrices où le chef de train se trouvera à côté du mécanicien; de plus, le gain de temps réalisé sur les trajets par suite de l'augmentalion de la vitesse commerciale des trains permeltra à chaque compagnie d'assurer l'ensemble de son service avec un personnel sensiblement réduit. Enfin, dans le cas où la traction sera assurée par plusieurs machines, une seule équipe suffira pour commander loules les locomolives et conduire le train.

Dans les dépôts, lés équipes chargées de l'entrelien (décrassage des foyers, nettoyage des tubes el des boîtes à fumée, allumage et surveillance des feux, etc...) pourront être supprimées

(1) L'importance de ces deux premiers atantages poura so trouver réduite par suite des progrès réalisés dans la culustruction des machines à vapeur et de l'augmentation de rendement qui en résultera. Par contre, ces progrès ne paraissent pas susceptibles d'avoir une influence sensible sur ce qui suit.

Ann. des P. et Ch., Memorres, 1923-VI. et. celles chargées de la revision périodique et des réparations, très réduites, les machines électriques ayant beaucoup plus rarement que les machines à vapeur besoin d'être revues ou réparées.

Par contre, un persomel nouvcau sera nécessaire pour surveiller le fonctionnement des postes de transformation, assurer l'entretien et la réparation de ces postes et de ces lignes. Mais il est très vraisemblable que l'effectif de ce personmel sera tris réduit et que l'électrification se traduira en définitive par une économie très sensible de main-d'œuvre.

d) La simplification dans la conduite des machines, d'où résultera une diminution de la fatigue imposée au persomnel et une plus grande facilité dapprentissage.

c) La puissance des locomotives à vapeur est actuellement. limitée par l'effort qu'il est possible de demander au chauffeur. Il existe, il est vrai, des pays dans lesquels l'on emploie des foyers à chargement aulomatique, mais ce procédé n'est pas en usage en France; il n'esl d'ailleurs pas sans présenter quelques inconvénients. Il est, au contraire, facile d'augmenter autant quón le désire, le nombre des essieux moleurs dans les trains ćlectriques et cela sans dépasser la limite de charge par essicu. Leffet de ces augmentations de puissance sera daulant plus sensible que déjà, à puissance brute égale, la locomolive électrique a sur la locomotive à vapeur l'avantage de procurer une puissance utile superieure, par suite de la disparition du poids mort que représente le tender et les approvisiommements en eau et charbon qu'il renferme.

La possibilité d'augmenter ainsi la puissance disponible permettra en particulier l'emploi pour les trains de voyageurs-d'un matèriel plus lourd, donc plus confortable.

f) La suppression de la fumée, d'où plus grande propreté des trains et économie d'entretien; cetle suppression sera particulièrement appréciable dans les grands lunnels dont la ventilation exigeait des installations assez compliquées et pas loujours très satisfaisantes; elle contribuera à augmenter la sécurité des transports, la fumée diminuant fréquemment la visibilité des signaux.

g) L'augmentation de la vitesse commerciale, due à l'accélération des démarrages et à la possibilité d'accroître très notablement la vilesse dans les mampes.

h) J'amélioration des conditions de transport (rapidilé, confortable, etc...) rendra les voyages plus faciles et plus agréables, ce qui entrainera sans aucun doute une ąugnentation du trafic.

i) I.e freinage électrique permeltra de récupérer dans les descentes une quantité notable d'énergic ; landis qu'actuellement le travail de la pesanteur dans les descenles est entièrement perdu sous forme de chaleur développée par le froltement des freins sur les roues, une certaine partie de ce travail pourra etre transformée en énergie électrique, les moleurs des locomotives cessant de jouer le rôle de moleurs pour devenir générateurs et envoyant du courant sur la ligne au lieu de lui en emprunter. Il en résultera une réduction de la quantilé d'énergie à fournir par les usines (1).

(1) Il ne laut cependant pas s'exagérer les bénéfices à retirer de la r'écupération; ce n'est que sur les lignes à fortes et longues pentes qu'elle permettra de produire une quantitó d'énergie importante; dans les régions où il n'existe que de faibles déclivités, le gain sera toujours médiocre et compensé en partie par le supplément de prix de revient des marchandises équipées en vue de cette récupération. Ceci est tellement vrai que la compagnio du Midi vient, à la suite des essais faits sur ses premières locomotives, de supprimer tout dispositil de récupération sur ses nouvelles machines en construction: 
Il n'est pas inutile de rappeler à ce sujet que la traction par courant continu offre, entre autres avantages, celui d'être le mode de traction avec lequel l'on sache actuellement le micux réaliser le freinage électrique par récupération à vitesse variable.

i) Les machines électriques étant en général symétriques, il n'est pas nécessaire de prévoir de dispositifs (plaque tournante, triangle en manceuvre), leur permettant de faire un demi-tour. De plus, comme il a élé dit plus haut, certains trains, les trains de banlieue par exemple, pourront être constitués par une rame de wagons encadréc de 2 automotrices, l'une en tête, l'autre en queue, d'où résultera une suppression de manceuvres de locomotives dans les gares terminus.

k) La possibilité de faire circuler des trains plus lourds ou plus rapides permettra d'augmenter le rendement des lignes de chemins de fer existantes el d'éviter dans certains cas, ou du moins de retarder, le doublement loujours très onéreux de voies qqui sont sur le point d'atteindre leur limite de capacité.

l) Les présomptions de responsabilité en cas d'incendie qui pèsent actuellement sur cerlaines compagnies (par exemple sur le Midi dans la traversée des Landes) se trouveronl supprimées.

m) Cerlaines lignes de chemins de fer présentent des pentes trop raides pour ètre actuellement accessibles aux trains lourds. On est obligé de faire subir un detour aux marchandises pour les eviler, d'où complication du service, augmentation des durées de trajet, etc... L'électrification de ces lignes, en rendant leur utilisation possible pour les trains de marchandises, supprimera ces inconvénients.

Tels sont les principaux avanlages que l'on est en droit d'attendre de l'électrification des chemins de fer.

Il ne faut d'ailleurs pas perdre de vue que, même dans le cas où cette électrification se traduirait par un bénéfice nul pour les compagnies, c'est-à-dire dans le cas où il serait absolument indifférent à ces compagnies d'électrifier ou non leurs réseaux, l'électrification n'en serait pas moins éminemment désirable au point de vue général, et cela pour trois raisons :

$1^{\circ}$ L'exécution des travaux d'aménagement considérables que nécessiterait cette électrification constituerait pour les entre- preneurs et les industriels français une source de marchés importants. Les compagnies dépenseraient les mêmes sommes pour le fonctionnement de leurs réseaux, mais une partie de ces sommes, au lieu d'aller rémunérer les entreprises minières et les armateurs étrangers, alimenterait notre industrie; on aperçoit immédiatement toutes les conséquences heureuses qui en découlent au point de vue national. C'est un point sur lequel on ne saurait trop insister.

$2^{\circ}$ Les lignes de transport d'énergie destinées à assurer cette électrification serviront, plus ou moins suivant les cas, mais toujours dans une mesure appréciable, à d'autres transports et aideront par là à la diffusion de l'électricité dans le pays.

30 Les lignes de transport étant placées sous le régime de la concession feront graluitement retour à l'Etat en fin de concsssion. Leur construction équivaut donc à un accroissement, un enrichissement du patrimoine national.

Ces trois raisons auxquelles, lorsque l'énergie sera de source hydraulique, il convient d'ajouter l'intérêt qu'il y a pour un pays déficitaire en charbon à réduire sa dépendance vis-à-vis de l'étranger, montrent que, même si l'électrification se traduisait par une légère augmentation de dépense, elle pourrait, au point de vue économique, n’être pas absurde.

En résumé, et ce sera ma conclusion : l'électrification des chemins de fer présente au point de vue national un intérêt incontestable, mais il faut se garder d'en conclure que toute électrification est bonne et que l'on peut électrifier à tort et à travers; il y a des lignes pour lesquelles l'électrification apparaît comme absolument contre-indiquée, d'autres pour lesquelles il semble qu'elle soit avantageuse, sans que l'on puisse d'ailleurs préciser l'importance de cet avantage tant en raison de l'instabillité des prix que de l'impossibilité de chiffrer certains bénéfices; dans les cas, d'ailleurs nombreux, où l'électrification des voies ferrées peut ètre raisonnablement envisagée à l'heure actuelle, elle se recommande moins par une économie immédiate et directe pour l'exploitant, économie généralement faible et toujours aléaloire, que par les avantages indirects très importants qui doivent en résulter. 